\title{
Predictive risk factors for sentinel lymph node metastasis using preoperative contrast-enhanced ultrasound in early-stage breast cancer patients
}

\author{
Jianghua Qiao ${ }^{1}$, Juntao $\mathrm{Li}^{1}$, Lina Wang ${ }^{1}$, Xiaoxia Guo ${ }^{2}$, Xiaolin Bian ${ }^{2}$, Zhenduo Lu ${ }^{1} \wedge$ \\ ${ }^{1}$ Department of Breast Surgery, Affiliated Cancer Hospital of Zhengzhou University (Henan Cancer Hospital), Zhengzhou, China; ${ }^{2}$ Department of \\ Ultrasound, Affiliated Cancer Hospital of Zhengzhou University (Henan Cancer Hospital), Zhengzhou, China \\ Contributions: (I) Conception and design: J Qiao, Z Lu; (II) Administrative support: None; (III) Provision of study materials or patients: J Qiao, Z Lu, \\ X Guo, X Bian; (IV) Collection and assembly of data: J Qiao, J Li, L Wang, Z Lu; (V) Data analysis and interpretation: J Qiao, J Li, L Wang; (VI) \\ Manuscript writing: All authors; (VII) Final approval of manuscript: All authors. \\ Correspondence to: Zhenduo Lu. Department of Breast Surgery, Affiliated Cancer Hospital of Zhengzhou University (Henan Cancer Hospital), \\ Zhengzhou 450008, China. Email: 13592596797@163.com.
}

Background Sentinel lymph node biopsy (SLNB) is the standard procedure for axillary staging in
clinically node-negative (cN0) breast cancer patients. The positive rate of SLNs in cN0 stage patients ranges
from $20.5 \%$ to $25.5 \%$, so identifying appropriate candidates for SLNB is quite challenging. The aims of
this study were to assess whether contrast-enhanced ultrasound (CEUS) could be utilized to noninvasively
predict SLN metastasis, and to explore the predictive value of the involved factors. Methods: Between May 2016 and May 2018, 217 consenting breast cancer patients undergoing SLNB were enrolled. Before the surgery, CEUS was utilized to identify the SLNs, and predict whether metastasis had occurred according to their enhancement pattern. Blue dye was also used to identify the SLNs during SLNB. The rates of identification and accuracy of both methods were recorded. The predictive outcomes of SLNs identified by CEUS were recorded and compared with the pathological diagnosis.

Results: Of the 217 cases, SLNs in 212 cases were successfully identified, comprising 208 cases identified by CEUS and 206 cases by blue dye, with no significant difference between the two methods $(\mathrm{P}=0.6470)$. A total of 78 cases were predicted SLN-positive preoperatively by CEUS, comprising 61 cases of SLN metastasis confirmed by pathology and 17 cases of no SLN metastasis, and 130 cases were predicted SLN-negative by CEUS, comprising 6 cases of SLN metastasis and 124 cases of no SLN metastasis. The sensitivity of CEUS preoperative prediction was $91.0 \%$, the specificity was $87.9 \%$, the positive and negative predictive values were $78.2 \%$ and $95.4 \%$, respectively, and the accuracy was $88.9 \%$. The maximum diameter size of positive SLNs predicted by CEUS was greater than that of negative SLNs (mean value $1.67 \pm 0.06 v s$. $1.40 \pm 0.05 \mathrm{~cm}, \mathrm{P}=0.0007)$. Similarly, the primary tumor size predicted SLN-positive by CEUS was greater than that in patients with negative SLNs (mean value $2.64 \pm 0.12$ vs. $1.79 \pm 0.09 \mathrm{~cm}, \mathrm{P}<0.0001$ ).

Conclusions: CEUS accurately identified SLNs and can be used to noninvasively predict SLN metastasis in early-stage breast cancer patients. However, the primary tumor size and the SLN size should not be overlooked by clinicians when judging the status of SLNs. This novel method may be a recommended strategy for identifying appropriate SLNB candidates.

Keywords: Breast cancer; contrast-enhanced ultrasound (CEUS); sentinel lymph node (SLN); sentinel lymph node biopsy (SLNB)

Submitted Nov 06, 2020. Accepted for publication Jan 14, 2021.

doi: $10.21037 / g s-20-867$

View this article at: http://dx.doi.org/10.21037/gs-20-867

^ ORCID: 0000-0001-6532-9761. 


\section{Introduction}

Axillary staging is a major survival predictor of breast cancer patients. The treatment protocols and prognosis of breast cancer are based on the axillary lymph node (ALN) status $(1,2)$. ALN dissection (ALND) has been the standard method of assessing ALN status (3), but had associated complications such as upper extremity dysfunction, lymphedema, paresthesia and pain, which can affect patients' quality of life $(4,5)$. Since the application of sentinel lymph node biopsy (SLNB) $(6,7)$, multiple studies have suggested that SLNB can accurately reflect ALN status $(8,9)$. Earlystage breast cancer patients who only underwent SLNB exhibited the same regional control, disease free survival and overall survival as patients who underwent ALND (10-12). Moreover, less complications occurred compared with the patients who underwent ALND (4,13). Hence, SLNB is recommended by the National Comprehensive Cancer Network (NCCN) guidelines as the standard procedure for axillary staging in clinically node-negative $(\mathrm{cN} 0)$ breast cancer patients (14).

Currently, patients with cN0 stage are usually diagnosed through physical examination, and conventional ultrasound and mammography (15). However, the criteria for identifying cN0 stage patients are controversial. The positive rate of SLNs in $\mathrm{cN} 0$ stage patients who undergo SLNB ranges from $20.5 \%$ to $25.5 \%(16,17)$. How to noninvasively identify appropriate candidates for SLNB is quite challenging. Although some studies attempted to demonstrate the feasibility of magnetic resonance imaging (MRI) to predict SLN metastasis in breast cancer $(18,19)$, there has not been consensus on the use of MRI or recommendation in the NCCN guidelines, because of the lack of evidence. The tracers used clinically to detect SLNs are at present blue dye, technetium-99m ( ${ }^{99 \mathrm{~m}} \mathrm{Tc}$ ) sulfur colloid, and indocyanine green fluorescence, but they cannot be used to judge the status of SLNs $(20,21)$ or to identify SLNB candidates.

Contrast-enhanced ultrasound (CEUS) is used to characterize lesions of according to the enhancement pattern of microbubble contrast agents (22). It is an established method to quantify hemodynamics and to diagnose hepatocellular carcinoma, prostate cancer, pancreatic tumors, and kidney disease (23-26). Other studies have demonstrated that CEUS is a feasible and safe method for the identification and localization of SLNs in breast cancer patients $(27,28)$. Additionally, some previous studies have indicated the value of the CEUS enhancement patterns for recognizing metastasized SLNs $(28,29)$. To our knowledge, there are few prospective studies assessing the accuracy of preoperative prediction of SLN metastasis utilizing CEUS and the related factors influencing the prediction.

In the present study, we focused on the feasibility and accuracy of predicting SLN metastasis utilizing CEUS and the value of the primary tumor and SLN sizes for predicting SLN metastasis and attempted to assess whether CEUS can be utilized to noninvasively predict SLN metastasis and identify appropriate SLNB candidates.

We present the following article in accordance with the MDAR checklist (available at http://dx.doi.org/10.21037/ gs-20-867).

\section{Methods}

\section{Patients}

This nonrandomized prospective study was performed in accordance with the Declaration of Helsinki (as revise in 2013) and approved by the Ethics Committee of the Affiliated Cancer Hospital of Zhengzhou University. All eligible patients were willing to undergo all study procedures and provided written informed consent prior to enrolment. The study was conducted between May 2016 and May 2018. Study participation was offered to women who met two criteria: (I) invasive breast carcinoma confirmed by histopathology from biopsy or resection specimen; and (II) clinically negative ALNs (cN0) through physical examination by two experienced breast surgeons (Jianghua Qiao and Zhenduo Lu) and conventional ultrasonography. The exclusion criteria were: (I) inflammatory breast cancer; (II) obviously enlarged ALNs or positive ALN confirmed by puncture pathology; (III) received neoadjuvant therapy previously; and (IV) undergone previous breast surgery or axillary surgery. We included a final cohort of 217 women (age range 29-72 years; mean age 47.5 years), all of whom had unilateral breast lesions.

\section{CEUS and image analysis}

Ultrasonography was performed with a Philips IU22 ultrasound system (Philips, The Netherlands) equipped with high-frequency linear array probes (L 5-12 Hz) and contrast pulse sequences. Sulfur hexafluoride microbubbles (Sonovue, Bracco Imaging, Italy) were used as the contrast agent. 
The CEUS examination and image analysis were performed by two experienced sonographers (Xiaoxia Guo and Xiaolin Bian), who were blinded to the patients' clinical data. The CEUS procedure was performed according to previous studies $(28,30)$. Briefly, after local anesthesia of the areola area, approximate $0.5 \mathrm{~mL}$ contrast agent was injected intradermally at 3, 6, 9, and 12 o'clock around the areola and the injection area was appropriately massaged for $15 \mathrm{~s}$. The ultrasonic probe tracked the enhanced lymphatic channels from the areola area to the axillary area. The first enhanced LNs were recorded as SLNs. Once identified, lymphatic channels and SLNs were marked on the skin surface, and the size and number of SLNs were recorded. If a lymphatic channel or LN was not detected successfully, the process was repeated.

There were three types of enhancement pattern of the SLNs: type I, homogeneous enhancement; type II, inhomogeneous enhancement, with hypoperfused or nonperfused area; and type III, weak or no enhancement (29). In this study, type I SLNs were identified as negative nodes, and type II or III SLNs were identified as positive nodes. When the enhancement pattern was inconsistent between the two sonographers, a consensus was reached through discussion.

\section{Surgical management of SLNs}

After signing the informed consent for surgery, a standard SLN biopsy was performed by two well-trained breast surgeons (Jianghua Qiao and Zhenduo Lu) according to previous studies (28-30). Briefly, after induction of general anesthesia, $0.5 \mathrm{~mL}$ methylene blue (Yookon, Beijing, China) was immediately injected intradermally in the periareolar upper outer quadrant region and 10 min later, an arc incision of the axilla was made along the lateral margin of the pectoralis major muscle. The blue-stained lymphatic channels were tracked to the axilla until the blue-stained LNs appeared. Their location, morphology and size were recorded in detail for comparison with the CEUS markers on the skin surface, and confirmation of the same LNs.

All the blue-stained, CEUS marked and suspicious LNs were excised and numbered according to the following categories: (I) both CEUS marked and blue-stained LNs (CEUS $/$ Blue $^{+}$); (II) only CEUS marked LNs (CEUS ${ }^{+} /$Blue $^{-}$); (III) only blue-stained LNs (CEUS/Blue ${ }^{+}$); (IV) neither CEUS marked nor blue-stained LNs (CEUS ${ }^{-} /$Blue $^{-}$). The SLNs of categories (I-III) were sent for frozen and paraffin pathological examination. The surgical procedure continued as standard ALND if the SLNs were proved positive. The patients in category (IV) underwent ALND immediately, regardless of pathology.

\section{Statistical analysis}

Statistical analysis was performed using Graphpad Prism software version 6.02 (Graphpad Software, Inc. La Jolla, CA, USA). Chi-square test or Fisher's exact test was used to compare the identification rate, which was defined as the proportion of patients with SLNs identified by either method. The independent samples $t$-test was used to compare means of continuous normal data. The diagnostic indices included sensitivity, specificity, positive-predictive value (PPV), negative predictive value (NPV), accuracy and the mean area under the receiver operating characteristic curves (AUC). All statistical tests were two-sided, and a $\mathrm{P}$ value $<0.05$ was considered statistically significant.

\section{Results}

\section{Clinicopathological characteristics}

A total of 217 patients with breast cancer were enrolled in the study (age 29-72 years, mean 47.5 years). Regarding menstrual status, 135 (62\%) patients were premenopausal, and $82(38 \%)$ patients were postmenopausal. The pathology, histologic grade, estrogen/progesterone receptor (ER/ PR) expression, human epidermal growth factor receptor-2 (HER-2) expression, molecular subtype of breast cancer, primary tumor size and SLN status are shown in Table 1. Standard ALND was performed in 72 cases, including 67 patients with positive SLNs and 5 patients with SLNs not found. ALN involvement was confirmed in 69 cases by paraffin pathology. According to the Tumor-Node-Metastasis (TNM)-based staging of breast cancers, 45 cases were categorized as N1 stage (1-3 ALNs with metastasis), 21 cases were N2 stage (4-9 ALNs with metastasis), and 3 cases were N3 stage ( $>9$ ALNs with metastasis).

\section{SLN detection}

Of the 217 cases, SLNs in 212 cases were successfully identified, comprising 208 cases identified by CEUS and 206 cases by blue dye. The identification rates of CEUS, blue dye, and the combined method were 95.9\% (208/217), $94.9 \%(206 / 217)$, and $97.7 \%(212 / 217)$, respectively $(\mathrm{P}=0.6470)$. Even though neither CEUS nor the blue dye 
Table 1 Characteristics of the primary tumor and SLNs $(n=217)$

\begin{tabular}{|c|c|}
\hline Characteristics & $\mathrm{N}(\%)$ \\
\hline \multicolumn{2}{|l|}{ Pathology } \\
\hline DCIS & $13(6.0)$ \\
\hline IDC & $193(88.9)$ \\
\hline ILC & $4(1.8)$ \\
\hline Other & $7(3.2)$ \\
\hline \multicolumn{2}{|l|}{ Histologic grade } \\
\hline G1 & $39(18.0)$ \\
\hline G2 & $136(62.7)$ \\
\hline G3 & $42(19.3)$ \\
\hline \multicolumn{2}{|l|}{ ER/PR expression } \\
\hline Negative & $66(30.4)$ \\
\hline Positive & $151(69.6)$ \\
\hline \multicolumn{2}{|l|}{ HER-2 expression } \\
\hline Negative & $162(74.7)$ \\
\hline Positive & $55(25.3)$ \\
\hline \multicolumn{2}{|l|}{ Molecular subtype } \\
\hline Luminal A & $31(14.3)$ \\
\hline Luminal B-HER2 (-) & $97(44.7)$ \\
\hline Luminal B-HER2 (+) & 26 (12.0) \\
\hline HER2 overexpression & $29(3.3)$ \\
\hline Basal-like & $34(15.7)$ \\
\hline \multicolumn{2}{|l|}{ Tumor size } \\
\hline Tis & $17(7.8)$ \\
\hline $\mathrm{T} 1$ & $171(78.8)$ \\
\hline $\mathrm{T} 2$ & $29(13.4)$ \\
\hline \multicolumn{2}{|l|}{ SLN status } \\
\hline Negative & $145(66.8)$ \\
\hline Positive & 67 (30.9) \\
\hline Not found & $5(2.3)$ \\
\hline
\end{tabular}

DCIS, ductal carcinoma in situ; ER/PR, estrogen/progestrone receptor; HER-2, human epidermal growth factor receptor-2; IDC, invasive ductal carcinoma; ILC, invasive lobular carcinoma; SLN, sentinel lymph node.

detected SLNs preoperatively in the remaining five cases, suspicious LNs were identified during SLNB, leading to immediate ALND regardless of pathology.

In total, 564 LNs were excised, consisting of 491 SLNs

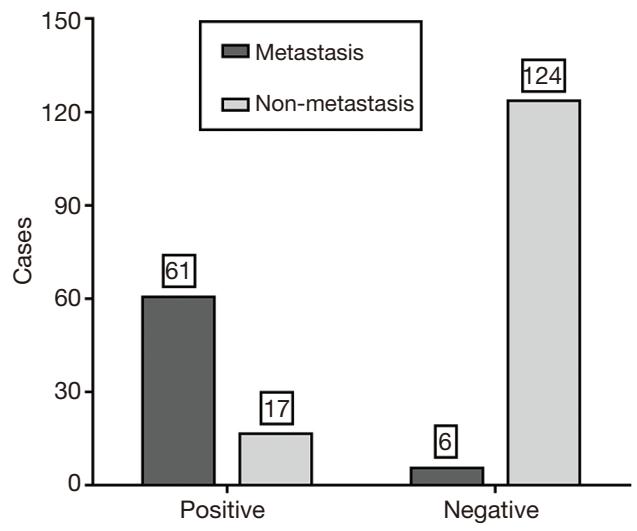

Figure 1 Contrast-enhanced ultrasound (CEUS) preoperative prediction and final sentinel lymph node (SLN) pathology.

and 73 non-SLNs. CEUS detected less SLNs than the blue-dye method (258 vs. 442). The mean number of SLNs detected by CEUS and blue dye was 1.2 and 2.1, respectively. According to the pathologic evaluation of SLNs, the positive rate of SLNs identified by CEUS was $38.0 \%$ (98/258), significantly higher than that of blue dye $(21.8 \%, 103 / 442)(\mathrm{P}<0.0001)$ (Table 2).

\section{Accuracy of CEUS preoperative prediction}

Pathologic evaluation of SLNs is the gold standard. Among the 208 patients identified by CEUS, 78 were predicted SLN-positive preoperatively by CEUS, comprising 61 patients $(78.2 \%)$ with SLN metastasis confirmed by pathology and 17 (21.8\%) without SLN metastasis, while 130 were predicted SLN-negative by CEUS, comprising 6 patients (4.6\%) with SLN metastasis and 124 (95.4\%) without SLN metastasis (Figure 1). The sensitivity, specificity, PPV, NPV, positive likelihood ratio $(+\mathrm{LR})$ and negative likelihood ratio (-LR) of CEUS preoperative prediction were $91.0 \%$ (61/67), 87.9\% (124/141), 78.2\% (61/78), 95.4\% (124/130), 7.55 and 0.10, respectively. The accuracy of CEUS preoperative prediction was $88.9 \%$ (185/208). The AUC was 0.895 (95\% confidence interval, 0.845-0.933).

\section{Effects of the size of the SLNs and primary tumor on CEUS preoperative prediction}

CEUS method detected 258 SLNs ultimately. A total of 107 SLNs were predicted positive preoperatively by CEUS, and 151 were predicted negative. The mean value 
Table 2 Classification of SLNs by CEUS and blue-dye method

\begin{tabular}{lccc}
\hline Classification & No. of cases (\%) & No. of lymph node (\%) & No. of positive SLNs \\
\hline CEUS $^{+} /$Blue $^{+}$ & $202(93.1)$ & $209(37.1)^{\mathrm{a}}$ & 95 \\
CEUS $^{+} /$Blue $^{-}$ & $6(2.8)$ & $49(8.7)^{\mathrm{a}}$ & 3 \\
CEUS $^{-} /$Blue $^{+}$ & $4(1.8)$ & $233(41.3)^{\mathrm{a}}$ & 8 \\
CEUS $^{-} /$Blue $^{-}$ & $5(2.3)$ & $73(12.9)^{\mathrm{b}}$ & 0 \\
Total & 217 & 564 & 106 \\
\hline
\end{tabular}

a, SLNs; ${ }^{\text {, }}$ non-SLNs. CEUS, contrast-enhanced ultrasonography; SLN, sentinel lymph node.
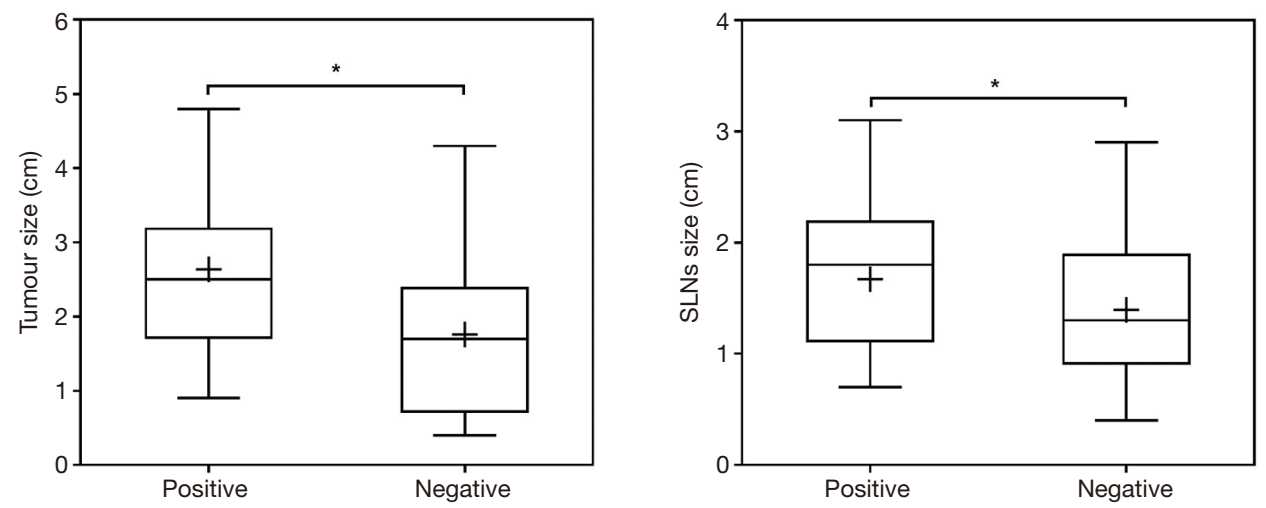

Figure 2 Effects of the size of (A) the primary tumor and (B) the sentinel lymph nodes (SLN) on preoperative prediction by contrastenhanced ultrasound.

of the SLN maximum diameter size was $1.67 \pm 0.06$ and $1.40 \pm 0.05 \mathrm{~cm}$, respectively, with a statistically significant difference $(\mathrm{P}=0.0007)$ (Figure 2). The primary tumor size was $0.5-4.8 \mathrm{~cm}$ (average $2.09 \mathrm{~cm}$ ). A total of 78 cases were predicted SLN-positive preoperatively by CEUS, and 130 were predicted SLN-negative. The mean value of the primary tumor size was $2.64 \pm 0.12$ and $1.79 \pm 0.09 \mathrm{~cm}$, respectively, with a statistically significant difference $(\mathrm{P}<0.0001)$ (Figure 2$)$.

\section{Discussion}

Axillary staging is the basis of breast cancer treatment protocols. For cN0 breast cancer patients, SLNB is recommended as the standard procedure for axillary staging (2). In general, patients with SLN pathological metastasis should undergo ALND, whereas the patients without metastasis do not need it. According to the updated American College of Surgeons Oncology Group (ACOSOG) Z0011 trial and the International Breast Cancer Study Group (IBCSG) 23-01 trial data, breast cancer patients with a T1 or T2 tumor and 1 or 2 positive SLNs who are treated with breast-conserving surgery, wholebreast radiotherapy and systemic therapy do not need to undergo ALND (31,32). However, the identification criteria for such patients are crucial. The trauma of breast cancer surgery is lessening but without a change in treatment outcome (33). The challenge is to noninvasively and accurately identify appropriate candidates for SLNB.

By comparing the identification of SLNs by CEUS and the blue-dye method, we found they detected $95.9 \%$ (208/217) and 94.9\% (206/217), respectively, with no significant difference. CEUS detected 258 SLNs, which was fewer than by the blue-dye method (442 SLNs). However, the positive rate of SLNs identified by CEUS was 38.0\% (98/258), significantly higher than with blue dye $(21.8 \%$, $103 / 442)$. CEUS is real-time imaging and can dynamically display the lymphatic vessels and enhanced LNs, which enables the SLNs to be easily identified and located (34). Although CEUS detected fewer SLNs than the blue-dye method, nevertheless, there was no significant difference 
between them in the identification rates. However, SLNB utilizing the CEUS method probably resulted in less physical trauma than the blue-dye method.

In addition to comparing the identification by CEUS and the blue-dye method, we also compared the predictive results for SLNs by CEUS and the pathological results of SLNB. We found that among the 208 patients identified by CEUS, 78 were predicted SLN-positive preoperatively by CEUS, comprising 61 (78.2\%) with SLN metastasis confirmed by pathology and 17 cases $(21.8 \%)$ without SLN metastasis. Of the 130 patients predicted SLN-negative by CEUS, 6 (4.6\%) had SLN metastasis and 124 (95.4\%) did not according to the pathologic diagnosis. These findings indicated that if the SLN-negative results predicted by CEUS were used as the treatment basis, approximately $4.6 \%$ of patients may be at risk of undertreatment because of the underestimation of SLNs pathology, which is much less that the $25.6 \%$ of patients diagnosed ALN-negative by conventional ultrasound in a previous study (17). Of the 6 cases of missed diagnosis, 4 were confirmed to have micrometastases by final histopathology (diameter $<2 \mathrm{~mm}$ ). Micrometastasis may not visibly destroy the normal structure of LNs, so would not show distinct changes in the CEUS enhancement pattern.

In clinical decision-making, whether the $\mathrm{LNs}$ are involved or not is judged mainly by sonographers, who are usually unfamiliar with the clinicopathological features that are widely used to indicate the malignancy of breast cancer. These features include menstrual status, pathological type, ER/PR and HER-2 status, Ki-67 index and the molecular subtype (35). Even though many studies have investigated the relationship between the clinicopathological features and SLN metastasis, which has value for clinicians $(16,36,37)$, they give limited assistance to sonographers in making clinical decisions. However, features including the size of SLNs and the primary tumor can be directly obtained through ultrasonography and are well known to sonographers. Hence, we attempted to determine whether these factors could affect clinical decision-making based on CEUS results. Unexpectedly, we found that the mean maximum diameter of positive SLNs detected by CEUS was greater than that of the negative SLNs, with a significant difference. Likewise, the primary tumor size predicted as SLN-positive preoperatively by CEUS was greater than in the negative SLNs cases, with a significant difference. To our knowledge, no publication has presented the relationship between SLN size, primary tumor size and SLN metastasis predicted by CEUS. The SLN size and primary tumor size should not be overlooked by sonographers in judging the SLN status.

According to the updated the NCCN Guidelines for Breast Cancer, SLNB has become the standard procedure for axillary staging in cN0 breast cancer patients (14). Others have tried to develop noninvasive or less invasive methods to replace SLNB, such as contrast-enhanced MRI and CEUSguided fine-needle aspiration or core biopsy $(18,38-40)$. However, further prospective studies with larger samples are still needed. We believe that SLNB is unlikely to be replaced by other noninvasive or less invasive methods at present because of the gold standard role of histopathology. Consequently, our aim was to assess whether CEUS could be utilized to noninvasively predict SLN metastasis and identify SLNB candidates rather than the feasibility of SLNB being replaced by CEUS. Our study showed that the sensitivity of preoperative prediction by CEUS was $91.0 \%$, the specificity was $87.9 \%$, the PPV and NPV were $78.2 \%$ and $95.4 \%$, respectively, and the accuracy was $88.9 \%$. Our findings are consistent with those from previous studies $(41,42)$. Although the accuracy and NPV are almost acceptable in clinical practice, SLN-negative results predicted by CEUS should not be the evidence for exemption from SLNB. Conversely, combining some factors such as SLN size and primary tumor size with CEUS predictive results may help identify the patients with SLN-positive results, who should undergo ALND directly with omission of SLNB.

In our study, we mainly recruited cN0 breast cancer patients, the criteria for which are controversial. This study might contain a slight selection bias. In addition, all the data for this study were from a single hospital. Further multicenter, randomized controlled and prospective studies with larger samples are required.

\section{Conclusions}

CEUS is an accurate method for SLN identification and can be used to noninvasively predict metastasis in earlystage breast cancer patients. However, the size of both the primary tumor size and the SLNs should not be overlooked by clinicians when judging the SLNs status. This novel method may be a recommended strategy for identifying appropriate SLNB candidates.

\section{Acknowledgments}

We thank all the personnel for their support and devoted work during data collection, and all the women who participated in the study for their fundamental contribution. 
The authors sincerely thank Xiaomi Fu, from the Department of Breast Surgery, Affiliated Cancer Hospital of Zhengzhou University, for her contribution to data collection.

Funding: This study was supported by Henan Provincial Medical Science and Technology Research Project, China (No. 201702250). The Sponsor had no role in the design and conduct of the study, collection, analysis, and interpretation of the data, preparation, review, and approval of the manuscript, or the decision to submit the manuscript for publication.

\section{Footnote}

Reporting Checklist: The authors have completed the MDAR checklist. Available at http://dx.doi.org/10.21037/gs-20-867

Data Sharing Statement: Available at http://dx.doi. org/10.21037/gs-20-867

Conflicts of Interest: All authors have completed the ICMJE uniform disclosure form (available at http://dx.doi. org/10.21037/gs-20-867). The authors have no conflicts of interest to declare.

Ethical Statement: The authors are accountable for all aspects of the work in ensuring that questions related to the accuracy or integrity of any part of the work are appropriately investigated and resolved. This study was performed in accordance with the Declaration of Helsinki (as revise in 2013) and approved by the Ethics Committee of the Affiliated Cancer Hospital of Zhengzhou University. All eligible patients were willing to undergo all study procedures and provided written informed consent prior to enrolment.

Open Access Statement: This is an Open Access article distributed in accordance with the Creative Commons Attribution-NonCommercial-NoDerivs 4.0 International License (CC BY-NC-ND 4.0), which permits the noncommercial replication and distribution of the article with the strict proviso that no changes or edits are made and the original work is properly cited (including links to both the formal publication through the relevant DOI and the license). See: https://creativecommons.org/licenses/by-nc-nd/4.0/.

\section{References}

1. Beek MA, Verheuvel NC, Luiten EJ, et al. Two decades of axillary management in breast cancer. Br J Surg 2015;102:1658-64.

2. Curigliano G, Burstein HJ, E PW, et al. De-escalating and escalating treatments for early-stage breast cancer: the St. Gallen International Expert Consensus Conference on the Primary Therapy of Early Breast Cancer 2017. Ann Oncol 2017;28:1700-12.

3. Barry JM, Weber WP, Sacchini V. The evolving role of axillary lymph node dissection in the modern era of breast cancer management. Surg Oncol 2012;21:143-5.

4. Mansel RE, Fallowfield L, Kissin M, et al. Randomized multicenter trial of sentinel node biopsy versus standard axillary treatment in operable breast cancer: the ALMANAC Trial. J Natl Cancer Inst 2006;98:599-609.

5. Hack TF, Cohen L, Katz J, et al. Physical and psychological morbidity after axillary lymph node dissection for breast cancer. J Clin Oncol 1999;17:143-9.

6. Jatoi I, Benson JR, Toi M. De-escalation of axillary surgery in early breast cancer. Lancet Oncol 2016;17:e430-41.

7. Veronesi U, Paganelli G, Galimberti V, et al. Sentinel-node biopsy to avoid axillary dissection in breast cancer with clinically negative lymph-nodes. Lancet 1997;349:1864-7.

8. Schrenk P, Wayand W. Sentinel-node biopsy in axillary lymph-node staging for patients with multicentric breast cancer. Lancet 2001;357:122.

9. McMasters KM, Tuttle TM, Carlson DJ, et al. Sentinel lymph node biopsy for breast cancer: a suitable alternative to routine axillary dissection in multi-institutional practice when optimal technique is used. J Clin Oncol 2000;18:2560-6.

10. Veronesi U, Paganelli G, Viale G, et al. A randomized comparison of sentinel-node biopsy with routine axillary dissection in breast cancer. N Engl J Med 2003;349:546-53.

11. Martelli G, Boracchi P, Ardoino I, et al. Axillary dissection versus no axillary dissection in older patients with T1N0 breast cancer: 15 -year results of a randomized controlled trial. Ann Surg 2012;256:920-4.

12. Agresti R, Martelli G, Sandri M, et al. Axillary lymph node dissection versus no dissection in patients with T1N0 breast cancer: a randomized clinical trial (INT09/98). Cancer 2014;120:885-93.

13. McLaughlin SA, Wright MJ, Morris KT, et al. Prevalence of lymphedema in women with breast cancer 5 years after sentinel lymph node biopsy or axillary dissection: objective measurements. J Clin Oncol 2008;26:5213-9.

14. NCCN Clinical Practice Guidelines in Oncology (NCCN Guidelines( $\left.{ }^{\circledR}\right)$ Breast Cancer. National Comprehensive Cancer Network; Version 1. 2018. Available online: 
https://www.nccn.org/professionals/physician_gls/pdf/ breast.pdf

15. Tucker NS, Cyr AE, Ademuyiwa FO, et al. Axillary Ultrasound Accurately Excludes Clinically Significant Lymph Node Disease in Patients With Early Stage Breast Cancer. Ann Surg 2016;264:1098-102.

16. Malter W, Hellmich M, Badian M, et al. Factors Predictive of Sentinel Lymph Node Involvement in Primary Breast Cancer. Anticancer Res 2018;38:3657-62.

17. Chen X, He Y, Wang J, et al. Feasibility of using negative ultrasonography results of axillary lymph nodes to predict sentinel lymph node metastasis in breast cancer patients. Cancer Med 2018;7:3066-72.

18. Liu C, Ding J, Spuhler K, et al. Preoperative prediction of sentinel lymph node metastasis in breast cancer by radiomic signatures from dynamic contrast-enhanced MRI. J Magn Reson Imaging 2019;49:131-40.

19. Dong Y, Feng Q, Yang W, et al. Preoperative prediction of sentinel lymph node metastasis in breast cancer based on radiomics of T2-weighted fat-suppression and diffusionweighted MRI. Eur Radiol 2018;28:582-91.

20. Shen S, Xu Q, Zhou Y, et al. Comparison of sentinel lymph node biopsy guided by blue dye with or without indocyanine green in early breast cancer. J Surg Oncol 2018;117:1841-7.

21. Papathemelis T, Jablonski E, Scharl A, et al. Sentinel Lymph Node Biopsy in Breast Cancer Patients by Means of Indocyanine Green Using the Karl Storz VITOM(R) Fluorescence Camera. Biomed Res Int 2018;2018:6251468.

22. Bar-Zion A, Yin M, Adam D, et al. Functional Flow Patterns and Static Blood Pooling in Tumors Revealed by Combined Contrast-Enhanced Ultrasound and Photoacoustic Imaging. Cancer Res 2016;76:4320-31.

23. Arita J, Takahashi M, Hata S, et al. Usefulness of contrastenhanced intraoperative ultrasound using Sonazoid in patients with hepatocellular carcinoma. Ann Surg 2011;254:992-9.

24. Wink M, Frauscher F, Cosgrove D, et al. Contrastenhanced ultrasound and prostate cancer; a multicentre European research coordination project. Eur Urol 2008;54:982-92.

25. Dietrich CF, Ignee A, Braden B, et al. Improved differentiation of pancreatic tumors using contrastenhanced endoscopic ultrasound. Clin Gastroenterol Hepatol 2008;6:590-7.e1.

26. Cao W, Cui S, Yang L, et al. Contrast-Enhanced Ultrasound for Assessing Renal Perfusion Impairment and Predicting Acute Kidney Injury to Chronic Kidney Disease
Progression. Antioxid Redox Signal 2017;27:1397-411.

27. Gkegkes ID, Iavazzo C. Contrast Enhanced Ultrasound (CEU) Using Microbubbles for Sentinel Lymph Node Biopsy in Breast Cancer: a Systematic Review. Acta Chir Belg 2015;115:212-8.

28. Xie F, Zhang D, Cheng L, et al. Intradermal microbubbles and contrast-enhanced ultrasound (CEUS) is a feasible approach for sentinel lymph node identification in earlystage breast cancer. World J Surg Oncol 2015;13:319.

29. Zhao J, Zhang J, Zhu QL, et al. The value of contrastenhanced ultrasound for sentinel lymph node identification and characterisation in pre-operative breast cancer patients: A prospective study. Eur Radiol 2018;28:1654-61.

30. Sever AR, Mills P, Jones SE, et al. Sentinel node identification using microbubbles and contrast-enhanced ultrasonography. Clin Radiol 2012;67:687-94.

31. Galimberti V, Cole BF, Viale G, et al. Axillary dissection versus no axillary dissection in patients with breast cancer and sentinel-node micrometastases (IBCSG 23-01): 10year follow-up of a randomised, controlled phase 3 trial. Lancet Oncol 2018;19:1385-93.

32. Giuliano AE, Ballman KV, McCall L, et al. Effect of Axillary Dissection vs. No Axillary Dissection on 10-Year Overall Survival Among Women With Invasive Breast Cancer and Sentinel Node Metastasis: The ACOSOG Z0011 (Alliance) Randomized Clinical Trial. JAMA 2017;318:918-26.

33. Livingston EH, Li HC. Breast Cancer Surgery: Less Is More. JAMA 2017;318:909-11.

34. Wang Y, Zhou W, Li C, et al. Variation of sentinel lymphatic channels (SLCs) and sentinel lymph nodes (SLNs) assessed by contrast-enhanced ultrasound (CEUS) in breast cancer patients. World J Surg Oncol 2017;15:127.

35. Carreño G, Del Casar JM, Corte MD, et al. Local recurrence after mastectomy for breast cancer: analysis of clinicopathological, biological and prognostic characteristics. Breast Cancer Res Treat 2007;102:61-73.

36. Orsaria P, Caredda E, Genova F, et al. Additional Nodal Disease Prediction in Breast Cancer with Sentinel Lymph Node Metastasis Based on Clinicopathological Features. Anticancer Res 2018;38:2109-17.

37. Ding J, Jiang L, Wu W. Predictive Value of Clinicopathological Characteristics for Sentinel Lymph Node Metastasis in Early Breast Cancer. Med Sci Monit 2017;23:4102-8.

38. Zhong J, Sun DS, Wei W, et al. Contrast-Enhanced Ultrasound-Guided Fine-Needle Aspiration for Sentinel Lymph Node Biopsy in Early-Stage Breast Cancer. 
Ultrasound Med Biol 2018;44:1371-8.

39. Cox K, Taylor-Phillips S, Sharma N, et al. Enhanced pre-operative axillary staging using intradermal microbubbles and contrast-enhanced ultrasound to detect and biopsy sentinel lymph nodes in breast cancer: a potential replacement for axillary surgery. Br J Radiol 2018;91:20170626.

40. Nielsen Moody A, Bull J, Culpan AM, et al. Preoperative sentinel lymph node identification, biopsy and localisation using contrast enhanced ultrasound (CEUS) in patients with breast cancer: a systematic review and meta-analysis. Clin Radiol 2017;72:959-71.

Cite this article as: Qiao J, Li J, Wang L, Guo X, Bian X, Lu Z. Predictive risk factors for sentinel lymph node metastasis using preoperative contrast-enhanced ultrasound in early-stage breast cancer patients. Gland Surg 2021;10(2):761-769. doi: 10.21037/ gs-20-867
41. Matsuzawa F, Omoto K, Einama T, et al. Accurate evaluation of axillary sentinel lymph node metastasis using contrast-enhanced ultrasonography with Sonazoid in breast cancer: a preliminary clinical trial. Springerplus 2015;4:509.

42. Zhang YX, Wang XM, Kang S, et al. Contrast-enhanced ultrasonography in qualitative diagnosis of sentinel lymph node metastasis in breast cancer: A meta-analysis. J Cancer Res Ther 2015;11:697-703.

(English Language Editor: K. Brown) 